\title{
8-Acetyl-labdanolic Acid
}

\section{(-)-(3S)-5-((1R,2R,4aS,8aS)-2-Acetoxy-2,5,5,8a-tetramethyldecahydro-1-naphthalenyl)-3- methylpentanoic Acid}

\section{Juan M. Castro, Sofia Salido, Joaquin Altarejos*, Manuel Nogueras and Adolfo Sanchez}

Departamento de Química Inorgánica y Orgánica, Facultad de Ciencias Experimentales, Universidad de Jaén, 23071 Jaén, Spain

Tel.: 34-953-002743, fax: 34-953-012141, E-mail: jaltare@ujaen.es

Received: 12 December 2001 / Accepted: 19 September 2002 / Published: 8 March 2003

Keywords: Diterpenes, labdanes, labdanolic acid, Cistus ladaniferus, acetylation, tertiary alcohol

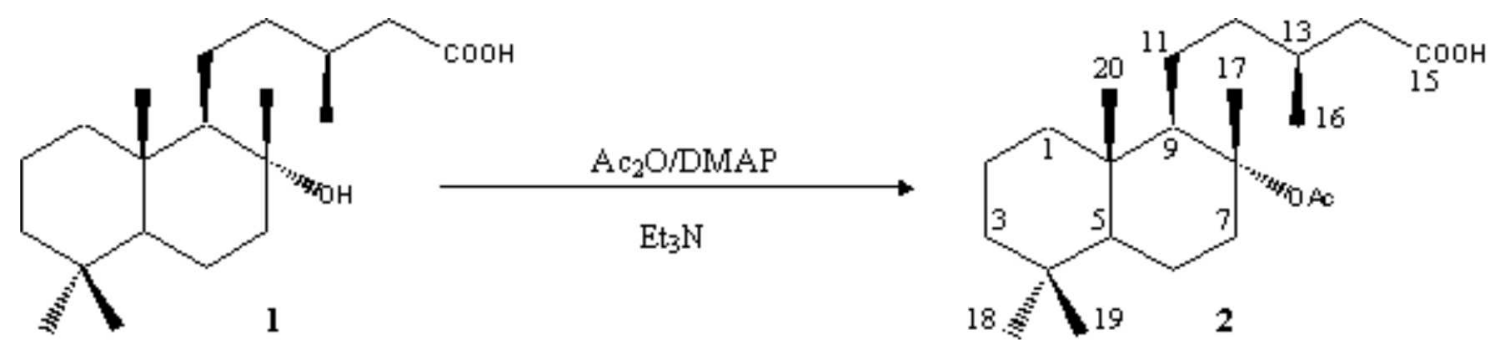

Acetic anhydride $(0.09 \mathrm{~mL}, 0.98 \mathrm{mmol})$ and $N, N$-dimethylaminopyridine $(2 \mathrm{mg}, 0.02 \mathrm{mmol})$ were added to a stirred solution of the alcohol $1(75 \mathrm{mg}, 0.23 \mathrm{mmol})$ in freshly distilled triethylamine $(1 \mathrm{~mL})$ [1]. The reaction was mantained between $35-40{ }^{\circ} \mathrm{C}$ for $72 \mathrm{~h}$ and, then, water was added $(10 \mathrm{~mL})$ and the mixture extracted with $\mathrm{Et}_{2} \mathrm{O}(3 \times 25 \mathrm{~mL})$. The combined organic layers were washed with $2 \mathrm{~N} \mathrm{HCl}(25 \mathrm{~mL})$ and brine $(3 \times 25 \mathrm{~mL})$. The organic phase was dried over anhydrous $\mathrm{Na}_{2} \mathrm{SO}_{4}$ and the solvent evaporated under reduced pressure to yield a residue $(77 \mathrm{mg}$ ) which was purified by flash chromatography on silica gel, using a 3:2 hexane/EtOAc mixture as eluent, to give the pure title compound 2 (55 $\mathrm{mg}, 0.15 \mathrm{mmol}, 65 \%$ ).

Mp: $131.0-132.8^{\circ} \mathrm{C}$ (white crystals, from hexane).

$[\mathrm{a}]_{\mathrm{D}}=-53.1^{\mathrm{o}}\left(\mathrm{c} 0.83 \mathrm{cg} \cdot \mathrm{mL}^{-1}, \mathrm{CHCl}_{3}\right)$.

IR (neat, $\left.\mathrm{n}, \mathrm{cm}^{-1}\right):$ 3400-2300, $1700(\mathrm{COOH}), 1732,1288,1188(\mathrm{OAc})$.

${ }^{1} \mathrm{H}$ NMR (300 MHz, CDCl 3 , d, ppm): $0.78\left(3 \mathrm{H}, \mathrm{s}, \mathrm{Me}_{\mathrm{b}}-4\right), 0.83(3 \mathrm{H}, \mathrm{s}, \mathrm{Me}-10), 0.86\left(3 \mathrm{H}, \mathrm{s}, \mathrm{Me}_{\mathrm{a}}-4\right), 0.98$ $(3 \mathrm{H}, \mathrm{d}, \mathrm{J}=6.6 \mathrm{~Hz}, \mathrm{Me}-13), 1.45$ (3H, s, Me-8), 1.92 (3H, s, OAc), 0.94-1.98 (16H, m, $\mathrm{H}-1,2,3,5,6,7 \mathrm{a}, 9,11,12,13), 2.16(1 \mathrm{H}, \mathrm{dd}, \mathrm{J}=15.0 \mathrm{~Hz}, 7.6 \mathrm{~Hz}, \mathrm{H}-14), 2.33$ (1H, dd, J=15.0 Hz, $6.6 \mathrm{~Hz}$, H'-14), 2.63 (1H, dt, J=12.7 Hz, $\left.3.5 \mathrm{~Hz}, \mathrm{Hb}^{-7}\right)$.

${ }^{13} \mathrm{C} \mathrm{NMR}\left(75 \mathrm{MHz}, \mathrm{CDCl}_{3}, \mathrm{~d}, \mathrm{ppm}\right): 39.50$ (C-1), 18.28 (C-2), 41.87 (C-3), 33.06 (C-4), 55.58 (C-5), 19.96 (C-6), 38.72 (C-7), 88.01 (C-8), 59.01 (C-9), 39.33 (C-10), 23.11 (C-11), 39.79 (C-12), 30.87 (C-13), 41.55 (C-14), 179.41 (C-15), 19.76 (C-16), 20.34 (C-17), 33.29 (C-18), 21.38 (C-19), 15.67 (C-20), 170.34 (OAc), 22.81 (OAc).

Acknowledgements: We wish to thank the Junta de Andalucía for financial support and the Ministerio de Educación, Cultura y Deporte for a Fellowship to J. M. Castro.

\section{References and Notes}


1. Urones, J. G.; Basabe, P.; Marcos, I. S.; González, J. L.; Jiménez, V.; Sexmero, M. J.; Lithgow, A. M. Ambergris Compounds from Labdanolic Acid. Tetrahedron 1992, 48, 9991-9998.

Sample availability: Available from the authors and from MDPI

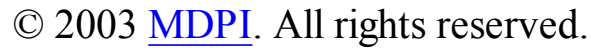

